\title{
Design and Implementation of an Expert Management System for Automobile Fault Detection
}

\author{
ABUBAKAR Dahiru Muhammed ${ }^{1}$, AJIBOLA Aminat. A $^{2}$, MUHAMMAD SANUSI ${ }^{3}$ \\ Research Scholar ${ }^{1}$, Lecturer $^{2-3}$ \\ ${ }^{1-3}$ Department of Computer Science, \\ University of Abuja, Abuja,
}

Nigeria

\begin{abstract}
A system has been developed to communicate with the On-Board Diagnostics system of a car using the Controller Area Network communication protocol. The system requests the stored trouble codes that might have been detected by the diagnostics system and sends them to a server. The information is accessed through a web interface. The web interface allows the user to find the solutions in the database together with the detected faults. The database also contains information about trouble codes, such as their symptoms, causes and how to fix them. Monitoring the communications while testing the prototype showed that the system works as intended and can communicate with cars from different manufacturers and extract the data. The prototype system was compared with a commercial scan tool and testing showed that they both produced the same results. The second module of the project deals with the design and implementation of an expert system for car faults diagnosis. The project is motivated by the need to guide car owners and learners' motor mechanics in the maintenance and troubleshooting of motor problems without having to resort to presumptions and conjectures. Particularly, it is expected that the proposed design would ensure that car owners have proper assistance in times of crisis and what's more, will save them from the clutches of exploitative roadside mechanics. For this purpose, a rule-based artificial intelligence (AI) technique was utilized to obtain theoretical and practical expert system parameters, and then a conceptual expert system was designed. The expert system functioning is based on the database of car faults, symptoms and their correction, which make up its knowledge base. The new system was developed using PHP and MySQL database. This language was chosen because of its easy syntax and features for developing web-based applications.

Key Words: Car Faults diagnosis, Electronic Control Units (ECU), Controller Area Network (CAN), On-Board Diagnostics $(O B D)$, Expert systems (ES), Artificial Intelligence (AI), Knowledge-Based System (KBS), Troubleshooting.
\end{abstract}

\section{INTRODUCTION}

Nowadays, the car technology is very crucial and significant to any car manufacturing companies as car specifications are changing rapidly stimulated by environmental and economic factors. This issue presents a challenge to both car mechanics and drivers in handling car fault and malfunctions. With the advent of new technologies like the hybrid engine, there are many changes that need to be learned. In fact, in a normal situation, car fault identification is still a challenging task, especially for the inexperienced mechanics and drivers.

Consequently, the success of finding the fault is extremely dependent on the expertise of the individual. However, the dependence on the experts can be minimized if the expertise is captured, documented, and retained in some computer applications

\section{BACKGROUND OF THE STUDY}

Today's vehicles have many built in computer systems that control parts of the car such as fuel injections, airbags or brakes. All of these systems are controlled by one of several Electronic Control Units (ECU) which communicate with each other over the internal high speed Controller Area Network (CAN) of the car. There is also a computer system called On-Board Diagnostics (OBD) that can discover and diagnose problems with the data reported by the ECUs. If a problem occurs, the OBD system generates a trouble code that which makes it possible for a service engineer to identify and fix the problem. Trouble codes and other diagnostic information can be accessed by plugging an OBD scan tool into the OBD interface in the car. 
The main advantage with having an OBD system is that it makes it easier to diagnose faults that occur in the vehicle. An advantage from a sustainability point of view is that vehicle emissions can be reduced by discovering and fixing problems, that makes the vehicles emission levels rise, that otherwise might not have shown any noticeable symptoms to the user or service personnel.

Also the second module which is Expert systems (ES) are a branch of artificial intelligence (AI), and were developed by the AI community in the mid-1960s. An expert system can be defined as "an intelligent computer program that uses knowledge and inference procedures to solve problems that are difficult enough to require significant human expertise for their solutions". We can infer from this definition that expertise can be transferred from a human to a computer and then stored in the computer in a suitable form that users can call upon the computer for specific advice as needed. Then the system can make inferences and arrive at a specific conclusion to give advices and explains, if necessary, the logic behind the advice. ES provide powerful and flexible means for obtaining solutions to a variety of problems that often cannot be dealt with by other, more traditional and orthodox methods. The terms expert system and knowledge-based system (KBS) are often used synonymously.

\section{OBJECTIVES OF THE STUDY}

The main objective of the research is to develop a system that can read trouble codes from an OBD system and make the information accessible to the user, while the second module is an Expert System. The aim of the second module proposed system is to provide quick and precise expert guidance to car fault diagnosis. Additionally, for training purposes, it helps in reducing the knowledge gap between different individuals in car fault diagnosis. The specific objectives of the research are as follows:

$>$ Setting up a web server and interface with a database containing OBD trouble code information from which users can read and make changes to the information in the database.

$>$ Developing a working communication channel that enables the system to extract relevant information from the car and send it to the screen.

$>$ To develop an ES that supports the implementation of the proposed system's functionality.

$>$ To provides facility in order to take the user opinion on any solutions disagree with the system's diagnosis?

\section{SCOPE OF THE STUDY}

The expert system may contain certain limits and flaws, as it is only a prototype. We commonly know that a car has many parts on its body and even in the body itself. However, the system will only deal with problems that are normally or commonly found in the engine part of the car as the system is just a prototype, which means the function will be expanded through time. The system will only deal with 3 cars models (Mercedece Benz, Toyota and Honda) produced from the year 2000 till date. Which will covers the following:

$>$ Repairs of Minor Faults in cars

$>$ Detecting the Cause of the problem

$>$ Suggest how to repair the car

\section{REVIEW OF RELATED LITERATURE}

Information gathering is mainly done by reading papers on related projects and International Organization for Standardization (ISO) standard documents. The ISO documents are not available for free, but contact with a company that develops OBD related software and hardware, Opus Group AB, made it possible to obtain information about the relevant ISO standards and access to the corresponding documents.

One of the earlier published references on Expert System (ES) is done by [7], whereas the first diagnostic ES for technical fault diagnosis was developed at MIT in the early 1970's as stated by [22]. Some researches that utilize the expert system for the purpose of diagnosing the failure of different machines are reviewed in the following paragraphs.

Another decision model for car fault diagnosis in which an ES is utilized to help inexperienced mechanics and drivers was proposed and designed. The model consists of inference engine, knowledge base, database, user interaction and adaptive mechanism. The Inference engine uses backward chaining as a result of a small number of outputs with many possible inputs. In addition, the adaptive mechanism is utilized in the user interaction section in order to receive feedback about system diagnosis result. The feedback results are stored in a database. The adaptive system then processes the stored data and extracts additional rules with the goal of improving the knowledge base [8]. 
Car failure detection KBS is proposed by [1]. In this system, car faults are divided into three states: Start-up state, Run-stable state and Movement-state. Shell Rule based expert system (CLIPS) with forward chaining inference engine is used in the implementation. CLIPS store's the knowledge in rules form, which has logic-based representation as well as the production rules. The system interacts with the user through an interface and gives the diagnosis result with illustration. The rule-based expert system contains 150 rules for car failure causes. However, improvement in the domain knowledge and applying adaptive technique for knowledge creation are required in such system.

In the work of [15], Heavy Duty Diesel Engines (HDDEs) diagnosis is proposed. HDDEs maintenance requires high technical skills and extensive experienced mechanics that are scarce. As a result, employing an expert system in such domain can be highly useful. The HDDE faults diagnosis ES is able to successfully detect malfunctions in the engines and give recommendation of corrective actions. System development leads to collection of valuable information related to HDDE fault diagnosis and training. However, updating the knowledge base affects the reasoning process performance especially in the continuous run. They propose Bayesian Network technology for modeling the inference engine of the HDDEs. The HDDEs faults diagnosis expert system has a high potential for rapid diagnosis revealed a hidden fault in the engine and re-recommendation proper procedures of corrective actions. The results show that employing an expert system in such domain is highly useful. Heavy-duty diesel engines require high skills and extensive experience in the field of mechanics. The limitation of the work is the simplicity of the knowledge base in which a very limited number of facts and rules are applied.

A research has been done by [23] to assist in the designing of an ES for car failure diagnosis and repair. Many factors are considered in this research such as the required time, the place and human expertise level. In addition, the ES development is accompanied by reviewing the technologies used in designing such system to conclude the best means to be followed. However, the proposed prototype is not promoted to be used as a complete application due to time and resources limitations. Thus, adopting new rules to be

Expert systems have been developed for a wide range of applications to perform reasoning tasks in diagnosis, control, and detection research fields [4]. The first expert system for technical fault diagnosis is developed at MIT in the early 1970's [22]. Recently, expert systems have been proposed for cars failure diagnoses. [5], propose a car automotive diagnostic model based on hybridization of an expert system and decision support system. The failure diagnosis is made by the expert system and the process of the replacement of a faulty component is made by the decision support system. This hybrid system is practically tested and the test results show that the system increases the efficiency of labor and reduces the mental workload of the workers.

In the work of [16], they model an expert system for cars failure diagnosis and repair. They propose a recursive inference engine that its applicable rules perform according to the phases of matching, selecting and executing. The knowledge base has a complex structure and contains a large number of facts and rules. The system takes into account many factors affecting the expert system such as the time and level of expertise. The prototype of this system is not promoted to be used as a complete application due to time and resource limitations.

Similarly, [18], present the imperatives for an expert system in developing car failure detection models. They address the problem of cars failure diagnosis and the need for applying the expert system in this field. Subsequently, they develop a Car Failure and Malfunction Diagnosis Assistance System (CFMDAS). The CFMDAS consists of three main parts which are the knowledge acquisition the graphical user interface and expert system. The expert system has a conventional forward chaining interface engine. The CFMDAS system is found to be highly useful in assisting mechanics for failure detection and training purposes.

A portable knowledge-based system for cars failure notification was also developed. The system consists of three main important parts: a knowledge base, an inference engine and a user interface which has a standard dashboard of a car. The inference engine has a mathematical model and works based on algebraic architecture. The system helps the driver to be aware of the dashboard icons conditions and meanings. The driver interacts with the system to identify the car conditions based on the dashboard lights indications. The system is developed as a mobile application to alarm the driver on a possible failure and helps the driver to handle the failure [6].

For the agent-based expert systems, there are several studies that integrated software agents in expert systems. The aim of the studies is to improve the quality and accuracy of the overall systems' performance [19]. The agent provides proactive capabilities and the multi-agent provides deliberative capabilities to the systems [20]. These capabilities make the systems feasible to analyze some possible interactions among modules at design time and hence efficiently handle complexities and anomalies [13]. The following are some examples of deploying agents in expert systems.

A framework of distributed multi-agent to improve the accuracy of an expert system was proposed. The agents interact with users to collect relevant information then combine it with the knowledge base data to discover a high order data or global knowledge. 
The framework includes data mining algorithms to retrieve and/or extract useful data of facts and rules from the knowledge base. The framework is applied in a knowledge-based medical diagnostic system for calculi disease [21].

Using a rule based approach a component expert system can be developed to carry out a through diagnosis of automobile engine faults, diagnosing the possible faults associated with each symptom and a correctional procedure for each of them. When we are talking of an expert system technology we are referring to a "computer software system that emulates the decision making ability of a human expert". It diagnosis problems and gives advice on what the cause of those problem are and can also give solution.

The expert system is one of the most important and active application areas of artificial intelligence which develops from 1960s [10]. It is an intelligent computer program based on knowledge, using expertise of expert to solve particular complex problems in certain fields [11]. After many years of development, a variety of expert systems have been emerged around various professional fields, they have been widely used and developed further in application [2]. It has several characteristics, including heuristic, transparency and flexibility [25],[26].

The expert system of automobile fault diagnosis can simulate diagnosis ideas of maintenance experts to find and remove automobile troubles [3]. The system mainly uses the build-in-vehicle sensors to obtain fault information of electronics control units. It makes use of artificial intelligence algorithm to process the obtained comprehensive fault information, analyses the causes of failures and feedbacks the analysis results to the users. The fault diagnostic objects include engine, chassis, electrical equipment and so on [24]. Fault diagnosis is an important application direction of artificial intelligence in automotive industry and it is the product of collaboration of field experts and engineers.

At present, there are several models for automobile fault diagnosis expert system, including rule-based diagnosis expert system, instance-based diagnosis expert system, behaviour-based diagnosis expert systems, fuzzy logic-based diagnosis expert systems and artificial neural network-based diagnosis expert system. Although the research of fault diagnosis expert system for automobiles has made some progresses but it still cannot fully replace the thought process of experts. It is necessary that the system should cooperate with experts in fault diagnosis field to obtain satisfactory diagnostic results.

The automobile intelligent fault diagnosis expert system is the demand and inevitable trend of the rapid development of automobile industry. Introducing the neural network technology into the automobile fault diagnosis expert system in recent years is an important development direction of automobile research and technology. From the theoretical study and practical application point of view, the fault diagnosis system based on neural network has unique advantages in knowledge acquisition, knowledge representation, reasoning, learning ability, fault tolerance and so on [12].

\section{METHODOLOGY}

The development of the Expert System on Car Engine Troubleshooting is based on the methodology that has been adopted from several existing methodologies for different applications especially in the field of computer science, software engineering, knowledge engineering and multimedia, since this expert system will be an integration of these technologies.

A detailed survey of expert systems was conducted and an observational methodology sometimes adopted. The method of factfinding called knowledge acquisition which is based on the Artificial Intelligence approach, to extract facts was also adopted. Interviews and research review was also adopted to extract facts for this study.

In the study and development of this expert system, the methods used for knowledge representation is Production System (production rule). In the production rule, there are one or more rules that are designed to solve one problem.

The research also depended on published and unpublished literatures on expert systems, intelligent knowledge based systems, troubleshooting and car engine dynamics when it becomes necessary from the internet. Finally, an implementation driven methodology was also employed to illustrate the software tool resulting from this study.

\section{SYSTEM DESIGN}

The analysis of the knowledge acquired in the knowledge acquisition phase had lead to the design of the system which includes the overall structure of the system's knowledge, the programming parts as well as the interfaces which is equally important. 
It was designed after the insight gained from the previous phase on the best approach for representing the expert's knowledge and problem solving strategies in the expert system beside that, the method that has been used to process the system's knowledge was also defined in this phase, where forward chaining rule based expert system was selected as the inference engine.

This decision was made after taking into consideration of the organization or architecture of the knowledge and the problem. Solving strategies determined earlier. After all of the elements have been designed, the development phase was started for both programming and the construction of the user interfaces.

Design phase was also considered as the phase of prototype development. In this project case, the final product itself is actually a prototype.

That means in the design phase, the partial prototype was built which represented the initial part of the full prototype expert system. The partial prototype was built for the purpose of providing a deeper insight and better understanding of the problem and system's requirements. In fact system's design is inherently an interactive process where finding from system testing are used to refine the system's knowledge and structure.

\section{a. On Board Diagnostics}

OBD is the computer system built into cars that monitors the performance of the engine components. It consists of several ECUs that use various sensors to collect data and evaluate the performance of the car. The OBD system will detect problems with the cars performance or functions before the problems become noticeable to the driver. A user can access the OBD on the car by connecting a scan tool to the OBD connector which allows the user to see diagnostic data such as detected faults or engine temperature.

OBD-I refers to the first generation of diagnostics that was developed during the 1980s where every vehicle manufacturer used different connectors and communication protocols due to a lack of standardization. OBD-II, or OBD2, is the successor to OBD-I and was defined in the early 1990s by the American organization Society of Automotive Engineers (SAE) and required all compliant vehicles to use a standardized connector, and one of several standardized communication protocols. OBD2 became a requirement for all cars sold in the USA in 1996 [14].

European On Board Diagnostics (EOBD) is the European version of car diagnostics and is technically equivalent to OBD2 but was not implemented until 2001 for petrol cars and 2004 for diesel cars. From here on, the term OBD2 will be used for OBD2 and EOBD specific information and OBD will be used as a general term for on board diagnostics

Many OBD standards were first defined by SAE and later adapted by ISO. This means that many OBD standards from SAE have a technically equivalent counterpart from ISO. Since ISO is an international organization while SAE is American, definitions from ISO are used from here on in cases where both of the SAE and ISO versions are technically equivalent.

OBD2 permits five different communication protocols, as listed in table 1, which can be used to communicate with the OBD2 interface. Most vehicle manufacturers only implement one of these protocols so it is often possible to identify the used communication protocol by looking at which pins are present on the connector.

Table 1: communication protocols in OBD2

\begin{tabular}{|l|l|}
\hline Standard & Description \\
\hline SAE J1850 & Pulse-Width Modulation (PWM) \\
\hline SAE J1850 & Variable Pulse Width (VPW) \\
\hline ISO 9141-2 & Similar to RS232 \\
\hline ISO 14230 & KeyWord Protocol 2000 (KWP2000) \\
\hline ISO 15765 & CAN (250kbps or 500kbps) \\
\hline
\end{tabular}

\section{i. Connector}


The OBD2 specification defines a standardized hardware interface to be used in OBD2 applications; the female ISO 15031-3 16pin connector. This connector is required to be reachable from the driver's seat and placed at most 2 feet $(0.61 \mathrm{~m})$ away from the steering wheel [27]. The pin-out of the connector is shown in figure 3.1 .

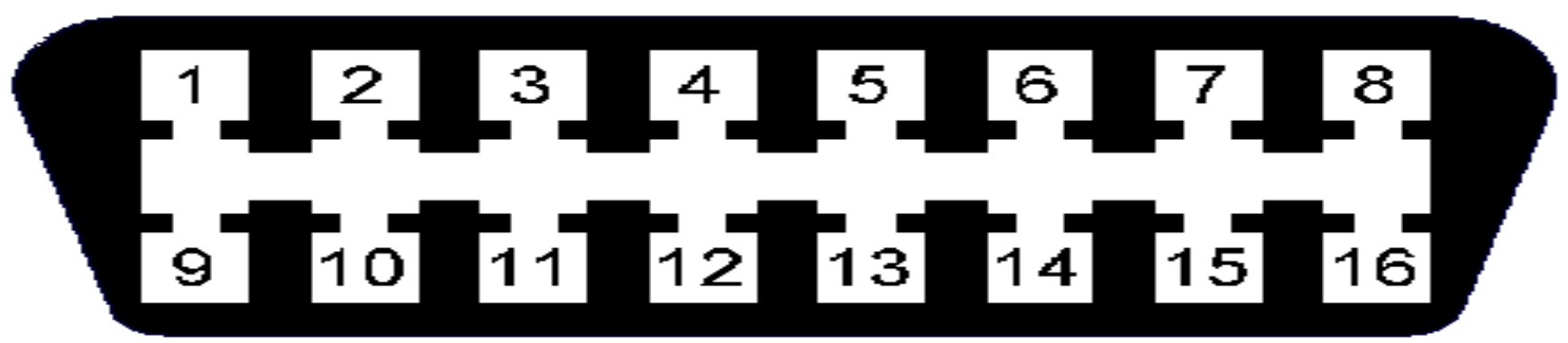

Figure 1: The OBD2 female connector

ISO 15031-3 defines the pin-out of the OBD2 connector according to table 2. Unspecified pins are left for manufacturer specific use. The connector was first defined in SAE J1962.

Table 2: Pin-out of the OBD2 connector according to ISO 15031-3

\begin{tabular}{|l|l|}
\hline 2: Positive line. PWM and VPW (SAE J1850) & 10: Negative line. PWM (SAE J1850) \\
\hline 4: Chassis ground & $14:$ CAN low (ISO 15765-4) \\
\hline 5: Signal ground & $15:$ L line (ISO 9141-2 and ISO 14230-4) \\
\hline 6: CAN high (ISO 15765-4) & 16: Battery voltage \\
\hline 7: K line (ISO 9141-2 and ISO 14230-4) & \\
\hline
\end{tabular}

\section{ii. Requests}

To issue a request to the OBD, specific codes need to be sent depending on the request. These codes are defined in ISO 15031-5 and consist of a mode of operation followed by a Parameter ID (PID). As of 2013, ten standard modes of operation are defined as listed below but vehicle manufacturers can define custom modes of operation outside of the ones defined in the standard. All vehicle manufacturers are required to at least support the emission related modes 01 (PID 00 and 01 ) 03 and 07 [17].

Standard modes of operation:

01. Show current data

02. Show freeze frame data

03. Show stored DTCs

04. Clear DTCs and stored values

05. Test results, oxygen sensor monitoring (non CAN only)

06. Test results, other component/system monitoring (Test results, oxygen sensor monitoring: CAN only)

07. Show pending DTCs (detected during current or last driving cycle)

08. Control operation of on-board component/system

09. Request vehicle information

0A. Show permanent DTC's (Cleared DTC's) 
For most modes there are several PIDs defined that specifies the request in more detail. For example mode 01, PID 0D requests the current vehicle speed and mode 09 PID 02 requests the VIN. Some modes do not require a PID, for example mode 03 requests the stored trouble codes and mode 04 clears them from memory.

Every PID has a defined response that is expected from the request. The responses are defined in ISO 15031-5 and describes in detail what the response should be, how many bytes the response contains and how the data is encoded in those bytes.

\section{iii. Trouble Codes}

Trouble codes are used to indicate the location of a malfunction in the car. When the OBD system recognizes a problem in the car, a trouble code is generated and saved in the ECU responsible for that subsystem. Many trouble codes are standardized and required but vehicle manufacturers are allowed to define their own. The structure of trouble codes and the definition of the standardized trouble codes are defined in ISO 15031-6 [9].

A trouble code consists of five characters as seen in table 3. The first digit is a character that describes which of the four main systems the fault belongs to. The second digit describes if the code is a generic code or a manufacturer specific code. The third digit describes the subsystem from which the fault originates. The fourth and fifth digits indicate the specific fault number.

Table 3: The structure of a trouble code

\begin{tabular}{|l|l|l|l|l|l|}
\hline $\begin{array}{l}\text { Character } \\
\text { Number }\end{array}$ & 1 & 2 & 3 & 4 & 5 \\
\hline Description & System & Control & Subsystem & ID & ID \\
\hline Allowed values & 'P' (Powertrain) & 0 (ISO controlled) & $0-\mathrm{F}$ & $0-\mathrm{F}$ & $0-\mathrm{F}$ \\
& 'C' (Chassis) & 1 (Manufacturer controlled) & & & \\
& 'B' (Body) & 2 (Reserved) & & & \\
& 'U' (Network) & 3 (Reserved) & & & \\
\hline
\end{tabular}

When trouble codes are sent by the OBD system as a reply to a mode 03 request, the trouble codes in the response are two bytes large and bitwise encoded according to table 4 . The first two bits describe the system character of the trouble code. The next two bits describe the control character. The remaining 12 bits describes the last three fields with four bits each and are on the form Binary Coded Decimal (BCD) [17].

Table 4: Trouble code encoding

\begin{tabular}{|l|l|l|l|l|l|}
\hline bits & $1-2$ & $3-4$ & $5-8$ & $9-12$ & $13-16$ \\
\hline Values & $00={ }^{\prime} \mathrm{P} '$ & $00=0$ & $0000=0$ & $0000=0$ & $0000=0$ \\
& $01={ }^{\prime} \mathrm{C} '$ & $01=1$ & $0001=1$ & $0001=1$ & $0001=1$ \\
& $10={ }^{\prime} \mathrm{B} '$ & $10=2$ & $\vdots$ & $\vdots$ & $\vdots$ \\
& $11={ }^{\prime} \mathrm{U} '$ & $11=3$ & $1111=\mathrm{F}$ & $1111=\mathrm{F}$ & $1111=\mathrm{F}$ \\
\hline
\end{tabular}




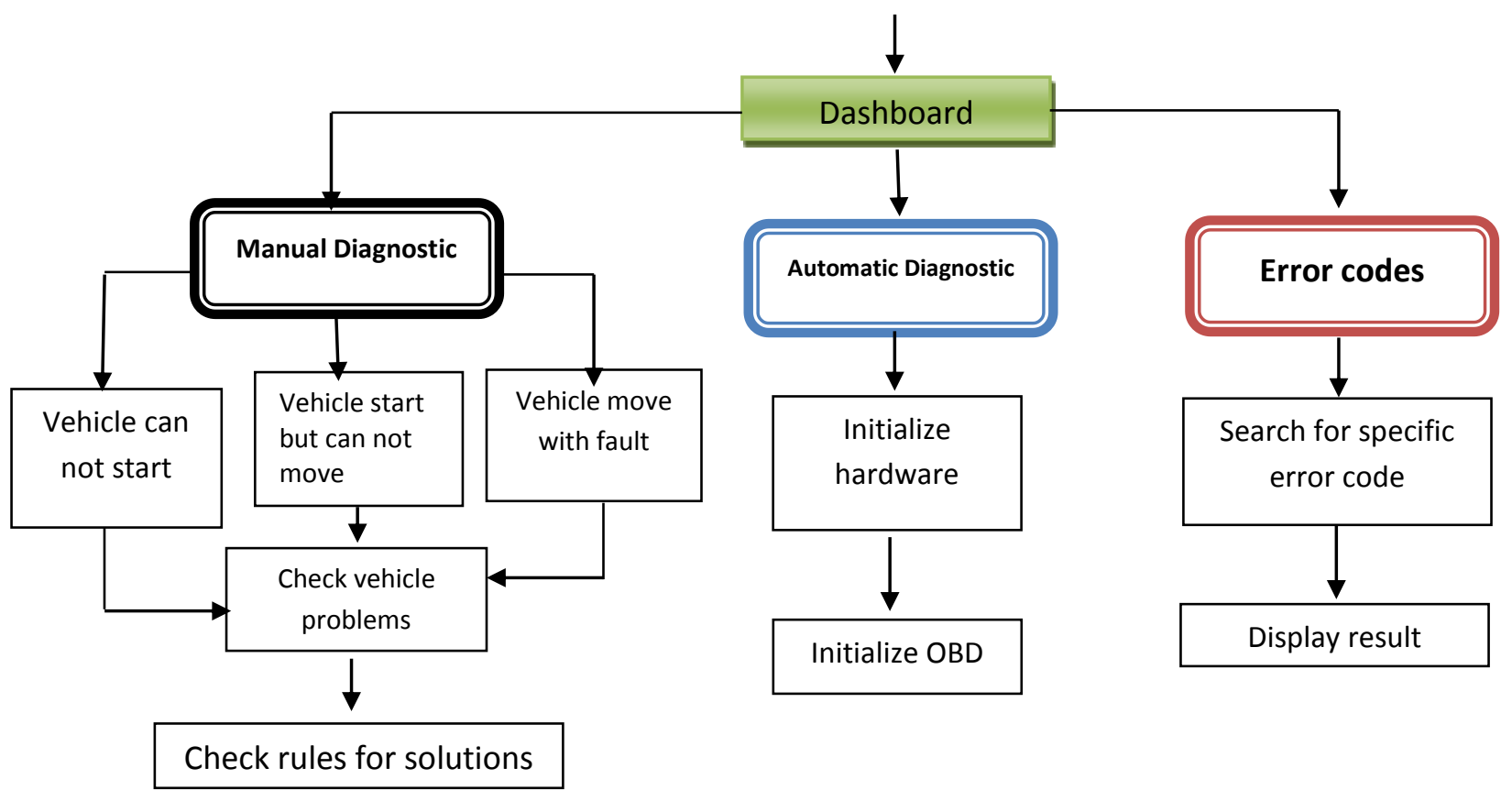

Figure 2: High Level Model of the Proposed System

\section{b. $\quad$ Rule Based Development Model}

The development of this expert system for car faults diagnosis was based on the current most popular choice of knowledge engineers for building an expert system that is rule - based expert system.

The popularity of this kind of system's development has grown due to the large numbers of successful rule- based system built and also abundance of rule- based expert software that is available easily. Besides, rule base expert system is a representation of the human beings natural reasoning and problem solving paradigm. It models human's production system using the following (Figure 3) modules.

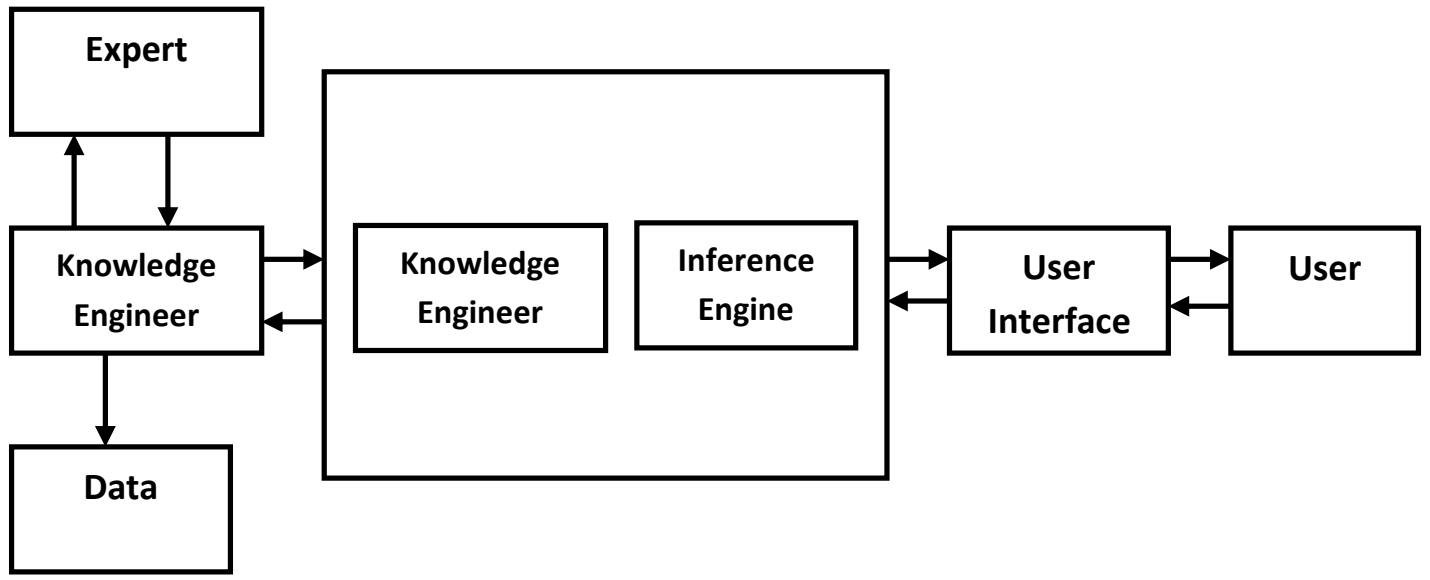

Figure 3: Structure of a Rule Base Expert System Model

$>$ Knowledge base models a human's long term memory as asset of rules.

$>$ Working memory- models a human's short term memory and contains problems facts both entered by the firing of the rules.

$>$ Inference engine- model human reasoning by combining problem facts contained in the working memory with rules contained in the knowledge base to infer new information.

Based on these concepts of reasoning which emulates the human problems solving strategies the expert system is structured accordingly to the fundamentals of it.

As used in the paradigm of problem - solving by this expert system for car faults diagnosis, the expert system has four basic elements which are adapted from the model of rule based system. Once the user logs in to the expert system, they will be asked questions which should be modelled according to the current problems. These new information will be entered to the working memory where the system will match this information with the knowledge contained in the knowledge base to infer new facts. 
Eventually the system and the conclusion is also entered into the working memory before displaying to the user through the interface. The four modules involved in the rule- based expert system are;

$>$ Knowledge base

$>$ Working memory

$>$ Inference engine

$>$ Interface

\section{SYSTEM IMPLEMENTATION}

The system consists of several key parts; the car, with an OBD interface, a module for communication to a database, the server hosting the database, and an interface.

\section{Program Structure}

The main task initializes the OBD communication and requests information from the connected car connects to the server and transmits the data. During every step it checks if everything works as expected, otherwise it raises a unique error code which is used by an error handler to identify the error and print a descriptive error text on the screen. If no error occurs the results is printed on the screen and the program allows you to fetch's the solutions from the database. The structure of the program is outlined in figure 4. A square with rounded corners indicates that a function is called that has its own flow chart in appendix.

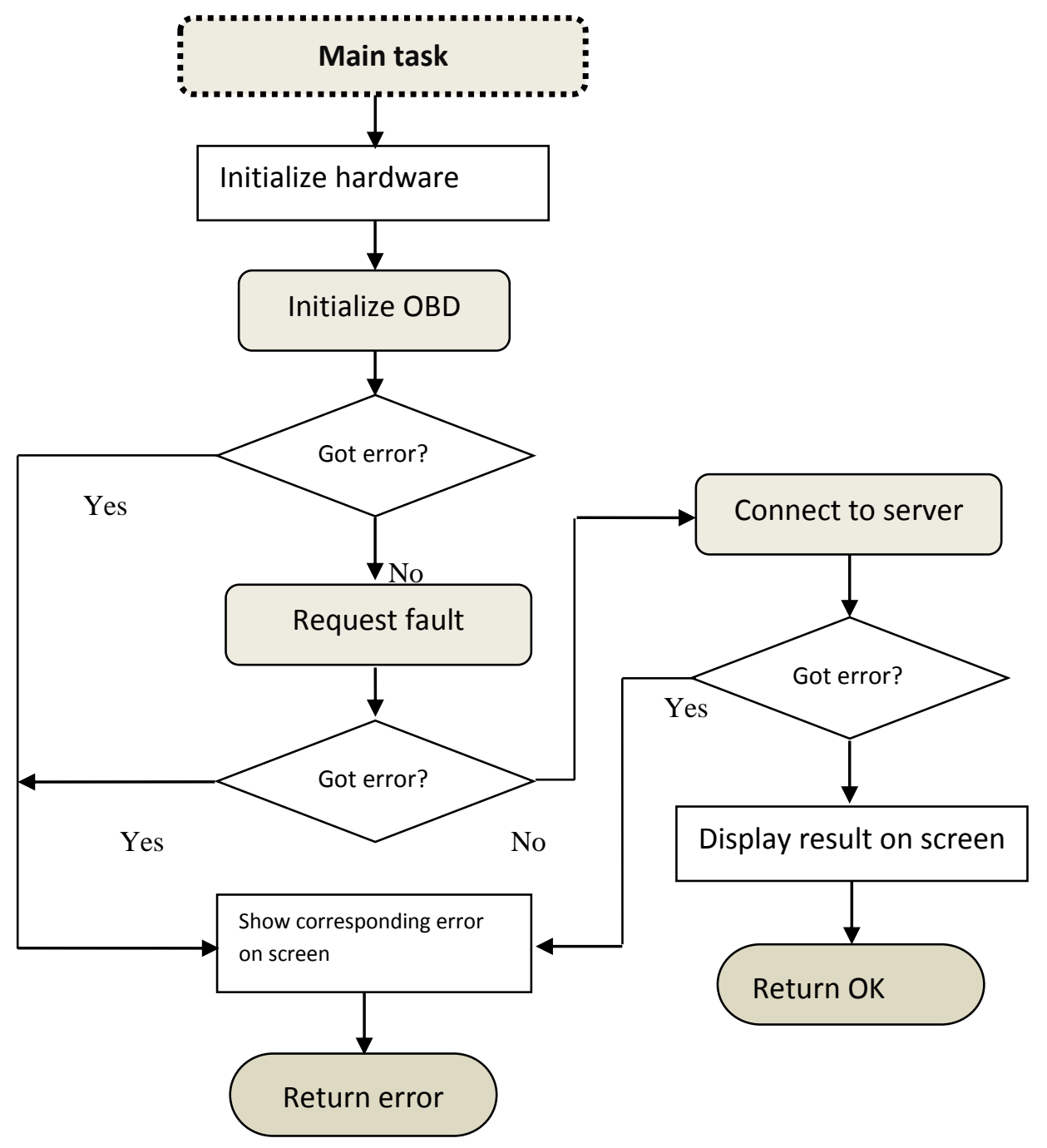

Figure 4: Flow chart of the Automatic Diagnostic main program

To keep the flow chart small and simple and not clutter the chart with non-relevant information, the display of runtime information on the SCREEN is not included in the chart. During the execution of the main program, appropriate information is shown on the SCREEN to indicate for example what module is initializing or when communicating with the server. 


\section{Web Interface Screenshot}

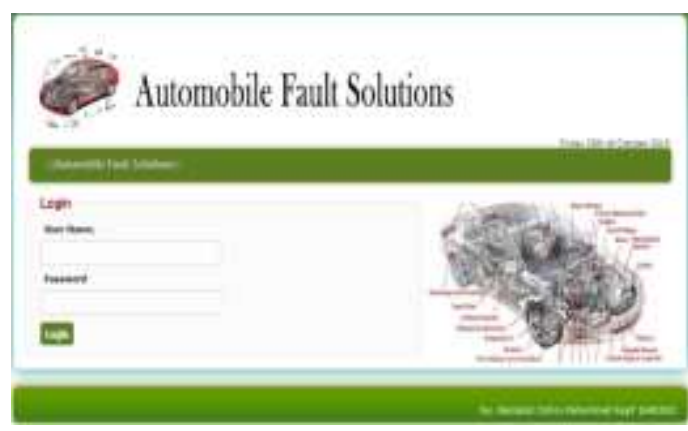

Figure 5: Login page

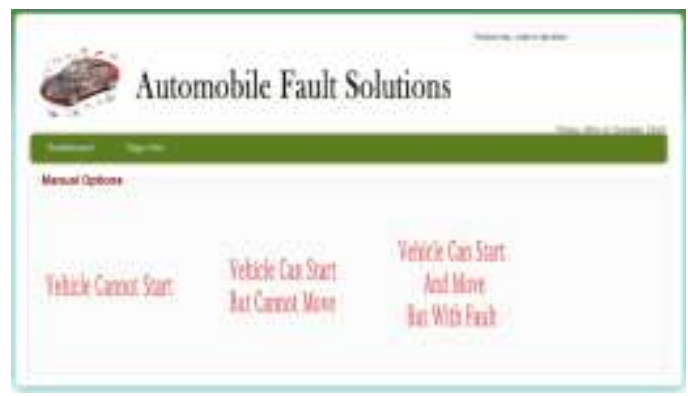

Figure 7: Expert System Menu page

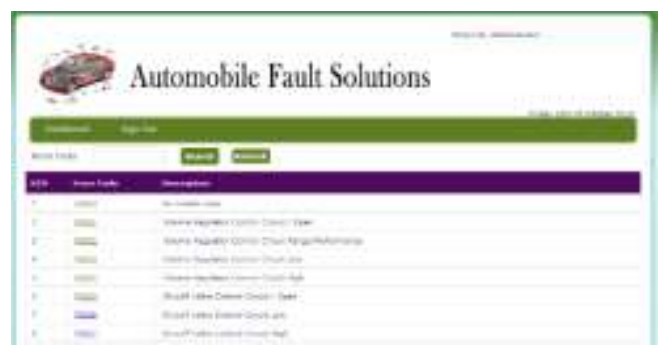

Figure 9: Trouble Codes page

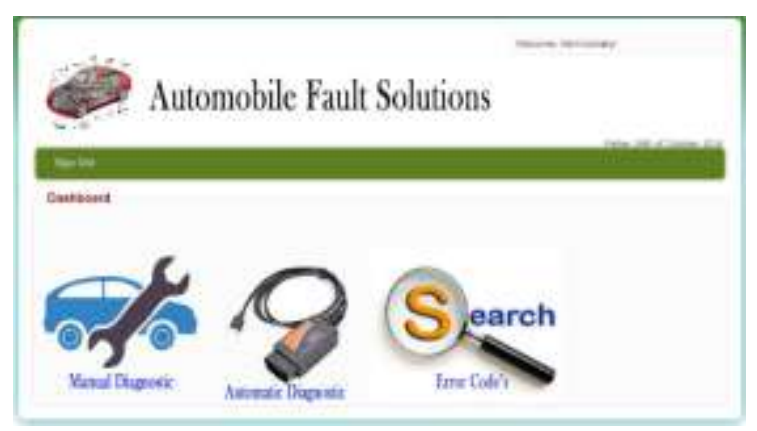

Figure 6: Home page

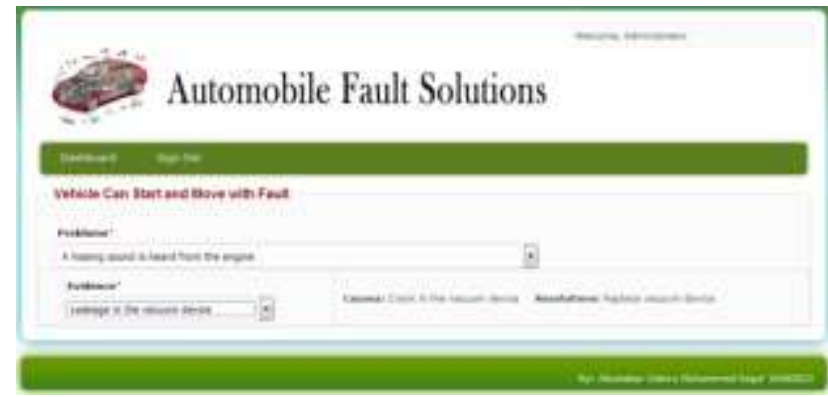

Figure 8: Expert Sytem Solution page

Automobile Fault Solutions

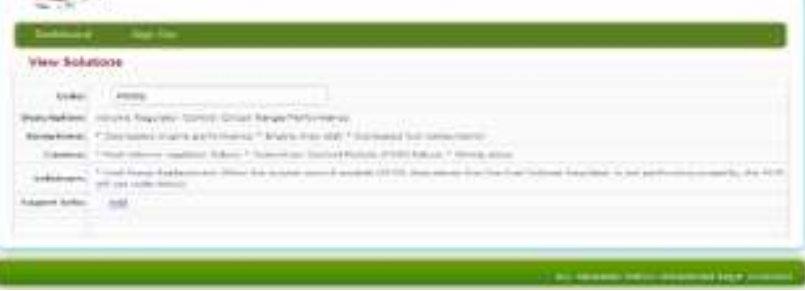

Figure 10: Error code solution page

\section{RECOMMENDATION}

The prototype of system was developed in a limited time and resources and thus it is not all that compatible and useful enough to be implemented in the really world yet. There must be so many other steps to be taken in refining the errors and rules before it can really be used in the real situation. When this is done, this expert system for car fault diagnosis would be ready to be use to assist all the car owners out there in situations where they have problems with their cars, so they can handle these problems themselves. Time and distance would no longer be constraints to them.

\section{CONCLUSION}

In this paper, a System for troubleshooting car engine faults was developed and implemented. The system was implemented using the PHP and MySQL programming language platform. During the test phase of the system it never gave wrong diagnosis according to the rules used. The system indicated that a full expert system will be practical and can be extremely useful in providing consistent car engine fault troubleshooting. Further work is needed to improve the system by adding sufficient domain knowledge that represents domain knowledge thoroughly. Plans are underway to convene experts to use the system to assist them in their jobs of car engine fault troubleshooting.

Preliminary validation of the program revealed that using OBD (scan tool) expert system to troubleshoot car engine problems is faster, accurate and more efficient than the manual approach.

The study has positively contributed to a culture whereby car users can begin to acquire a level of knowledge in the comfort of their homes and or offices, through the computer program developed in this study, and are able to resolve certain car problems. 
Finally, the study is significance as it is a pioneering effort, geared towards introducing a new area of application for expert systems. Furthermore, the prototype developed in this study is original, and can help other researchers carrying out further studies in this direction. it is believed that this effort will generate further research efforts in this direction, especially to have the implementation of a complete car troubleshooting expert system and further work is also needed to improve the system by adding sufficient domain knowledge that represents domain knowledge thoroughly.

\section{REFERENCES}

[1] A. T. Al-Taani, “An expert system for car failure diagnosis”, World Academy of Science, Engineering and Technology, Vol. 1, No. 12, pp. 4-7, 2005.

[2] Cao, X.L., 2004. Study of fault diagnosis techniques of intelligent roller hydraulic system based on expert system. Master Thesis, Chang'an University, Xi'an, China.

[3] Chen, C.Y., D.S. Zhang and P.H. Ren, 2003. Present state and perspectives of automotive fault diagnosis expert system. Chin. J. Mech. Eng., 39: 1-6.

[4] C. Angeli, 'Diagnostic expert systems: From expert's knowledge to real-time systems," in Advanced Knowledge Based Systems: Model, Applications \& Research, vol. 1, P. Sajja and R. Akerkar, Eds. TMRF e-Book, 2010, pp. $50 \_73$. [Online]. Available: http://www.tmr_ndia.org/eseries/ebook.html\#content

[5] D. D. Milanović, M. Misita, D. Tadic, and D. L. Milanović, "The design of hybrid system for servicing process support in small businesses," FME Trans., vol. 38, no. 3, pp. 143-149, 2010.

[6] E. Roanes-Lozano, J. L. Galán-García, and G. Aguilera-Venegas, “Aportable knowledge-based system for car breakdown evaluation," Appl. Math. Comput., vol. 267, pp. 758-770, Sep. 2015.

[7] E. Feigenbaum, Handbook of Artificial Intelligence: Heuris Tech Press, W. Kaufman Inc, 1981.

[8] K. S. E. Ricardo Nurzal, “A Decision Support System for Car Fault Diagnosis Using Expert System”, International Journal of Information Sciences for Decision Making, N 2, 1998.

[9] Kvaser Leaf Light HS OBDII. Kvaser AB, 2013 [viewed at 2013-11-22]. Available from http://www.kvaser.com/datasheets/kvaser_datasheet.php?ean=73-30130-00402-3

[10] Liao, S.H., 2005. Expert system methodologies and applications-a decade review from 1995 to 2004. Expert Syst. Appl., 28: 93-103.

[11] Liu, S.C. and S.Y. Liu, 2003. An efficient expert system for machine fault diagnosis. Int. J. Adv. Manuf. Technol., 21: 691-698.

[12] Men, Y.Z. and F.L. Wang, 2007. Research on neural network integration fusion method and application on the fault diagnosis of automotive engine. J. Mach. Des., 24: 64-65.

[13] O. Arsene, I. Dumitrache, and I. Mihu, “'Expert system for medicine diagnosis using software agents," Expert Syst. Appl., vol. 42, no. 4, pp. 1825_1834, 2015.

[14] On Board Diagnostics (OBD) | US EPA. U.S. Environmental Protection Agency, 2013 [viewed at 2013-11-22]. Available from http://www.epa.gov/obd/

[15] P. Nabende and T. Wanyama, "An expert system for diagnosing heavy-duty diesel engine faults," in Advances in Computer and Information Sciences and Engineering. Dordrecht, The etherlands: Springer, 2008, pp. 384-389.

[16] R. Aggarwal, S. Jain, Y. Jindal, and N. Verma, “Approach towards car failure diagnosis-an expert system," Int. J. Comput. Appl., vol. 1, no. 23, pp. 61-64, 2010.

[17] Road vehicles -- Communication between vehicle and external equipment for emissions related diagnostics -- Part 5: Emissions-related diagnostic services. ISO 15031-5:2011. Geneva, Switzerland : ISO.

[18] S. A. Mostafa, M. S. Ahmad, M. A. Mohammed, and O. I. Obaid, “Implementing an expert diagnostic assistance system for car failure and malfunction," Int. J. Comput. Sci. Issues, vol. 9, no. 2, pp. 1-7, 2012.

[19] S. A. Gutierrez and J. W. Branch, “A comparison between expert systems and autonomic computing plus mobile agent approaches for fault management," Dyna, vol. 78, no. 168, pp. 173-180, 2011. 
[20] S. A. Mostafa, M. S. Ahmad, A. Mustapha, and M. A. Mohammed, “A concise overview of software agent research, modeling, and development," Softw. Eng., vol. 5, no. 1, pp. 8_25, 2017.

[21] S. Sridhar, "Improving diagnostic accuracy using agent-based distributed data mining system," Inform. Health Soc. Care, vol. 38, no. 3, pp. 182_195, 2013.

[22] W. T. Scherer and C. C. White, III, "A survey of expert systems for equipment maintenance and diagnostics," in Knowledge-Based System Diagnosis, Supervision, and Control. NewYork,NY, USA: Springer, 1989, pp. $285-300$.

[23] Y. Jindal, S. Jain, R. Aggarwal and N. Verma, "Approach towards Car Failure Diagnosis-An Expert System", International Journal of Computer Applications (0975 - 8887) Volume 1 - No. 23, 2010.

[24] Yue, Y.J., X. Li and Q. Zong, 2011. Development of automobile fault diagnosis expert system based on fault Tree-neural network ensamble. Proceedings of the International Conference on Electronics, Communications and Control, September 9-11, 2011, Ningbo, China, pp: 2028-2031.

[25] Zhang, Q.W. and Y. Zhang, 2010. The design of an expert system on diagnosing the malfunction of hydraulic excavator. China Modern Educ. Equipment, 15: 58-60.

[26] Zhang, W., 2011. Based on expert systems in the automotive engine fault diagnosis application. Master Thesis, Taiyuan University of Technology, Taiyuan, China.

\section{APPENDIX: Flowcharts}

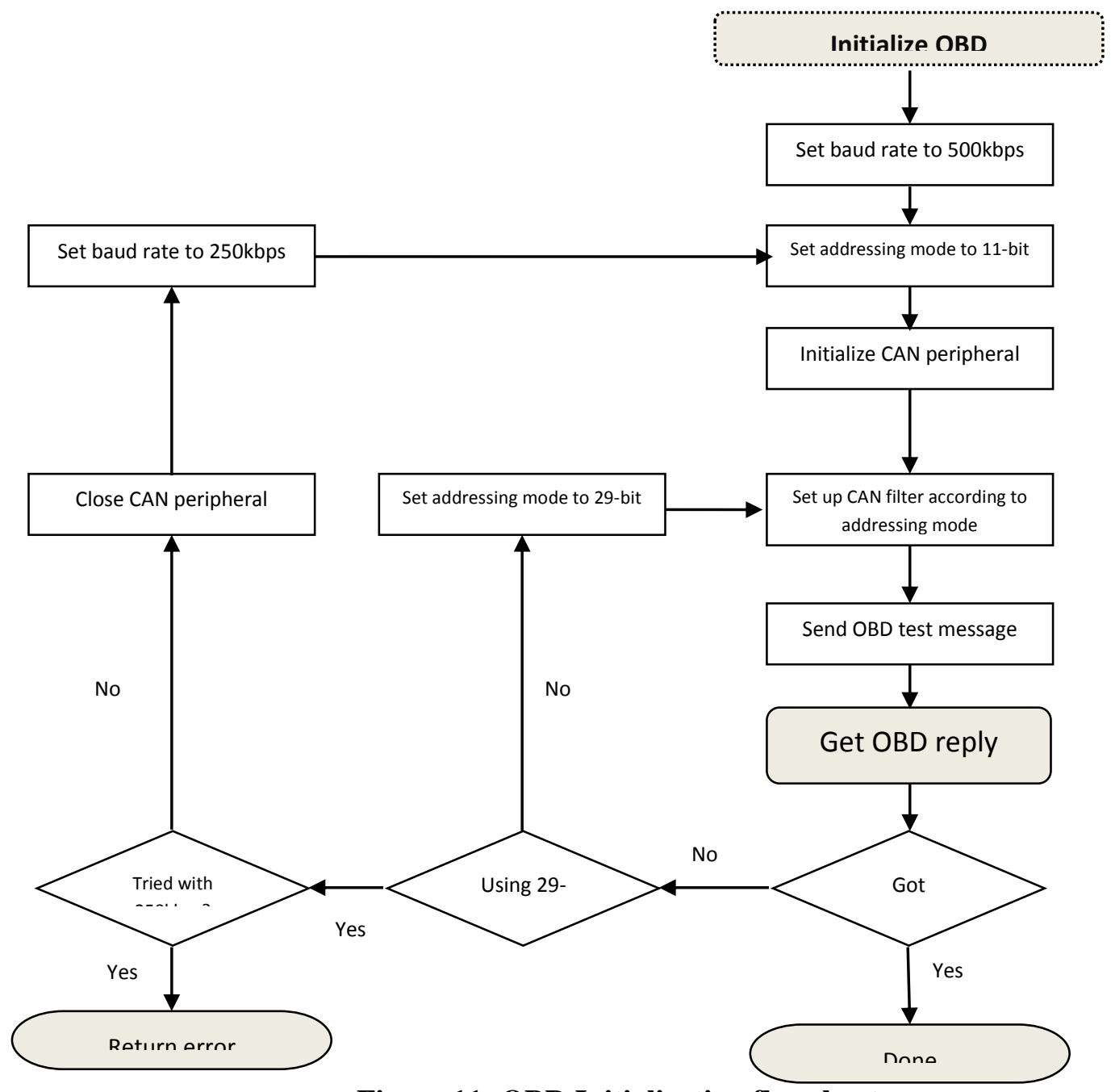

Figure 11: OBD Initialization flowchart

Check CAN message queue 
Figure 12: Get OBD reply flowchart

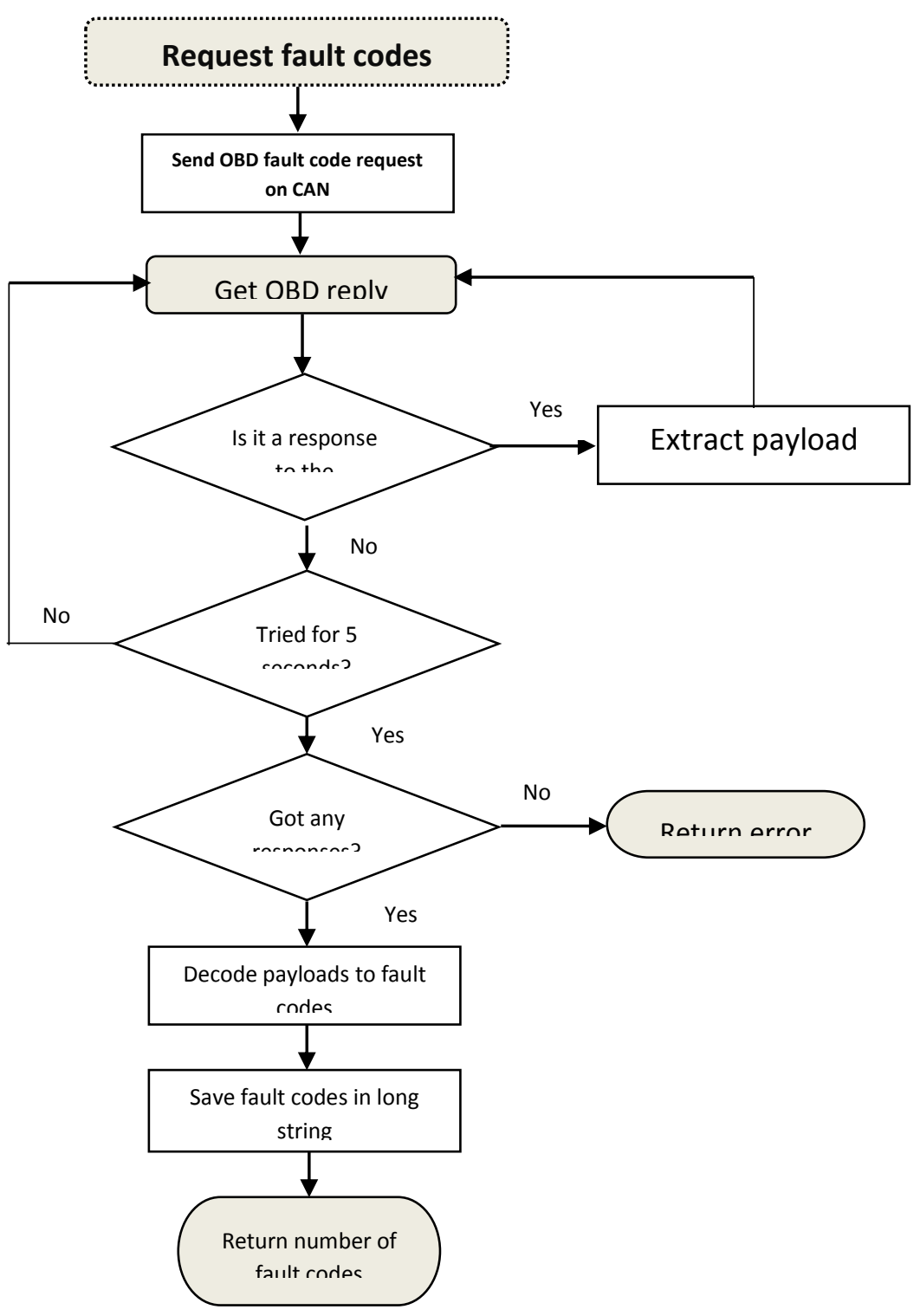

Figure 13: Fault code request flowchart 